\title{
Interaction Forces Between Pegylated Star-Shaped Polymers at Mica Surfaces
}

\author{
Xavier Banquy ${ }^{1}$, Frantz Le Dévédec ${ }^{2} \dagger$, Hsiu-Wei Cheng ${ }^{3,4}$, Jimmy Faivre ${ }^{1}$, Julian X.X. Zhu ${ }^{2}$, \\ Markus Valtiner ${ }^{3,4}$
}

${ }^{1}$ Faculty of Pharmacy, Université de Montréal, C.P. 6128, Succursale Centre-ville, Montréal, QC H3C 3J7, Canada

${ }^{2}$ Department of Chemistry, Université de Montréal, C.P. 6128, Succursale Centre-ville, Montréal, QC H3C 3J7, Canada

${ }^{3}$ Interface Chemistry and Surface Engineering Max-Planck-Institut fír Eisenforschung MaxPlanck-Straße 1, 40237 Dísseldorf (Germany)

${ }^{4}$ Institut fír physikalische Chemie, TU Bergakademie Freiberg Leipziger Straße 29, 09599 Freiberg (Germany)

KEYWORDS cholic acid, Pegylated polymers, dendronized brushes, surface forces, hydration layer

corresponding author: Xavier Banquy; email: xavier.banquy@umontreal.ca 
ABSTRACT We present a study focused on characterizing the interaction forces between mica surfaces across solutions containing star-shaped polymers with cationic ends. Using the Surface Forces Apparatus, we show that the interaction forces in pure water between surfaces covered with the polymer can be adequately described by the dendronized brush model. In that framework, our experimental data suggest that the number of branches adsorbed at the surface decreases as the concentration of polymer in the adsorbing solution increases. The onset of interaction was also shown to increase with the concentration of polymer in solution up to distances much larger that the contour length of the polymer suggesting that the nanostructure of the polymer film is significantly different from a monolayer. High compression of the polymer film adsorbed at low polymer concentration revealed the appearance of a highly structured hydration layer underneath the polymer layer. The results support that charged polymer chains do not necessarily come into close contact with the surface even if strong electrostatic interaction is present. Altogether, our results provide a comprehensive understanding of the interfacial behavior of star-shaped polymers and revealed the unexpected role of hydration water in the control of the polymer conformation. 


\section{Introduction}

Modification of interfacial properties of surfaces with adsorbed polymers is a well established strategy used in many different application fields. ${ }^{1}$ Several decades of research have shown that polymer conformation at surfaces is an essential parameter controlling interfacial properties such as wettability, ${ }^{2-3}$ friction, ${ }^{4}$ adhesion, ${ }^{5}$ topography. ${ }^{6}$ Polymer conformation can be tuned in many different ways using surface chemistry or polymer chemistry. For example, grafting of polymer chains at a surface can produce strongly attached polymer brushes, loops or even gels. ${ }^{7}$ The polymer composition can been modified to introduce stimuli responsive moieties to obtain instructive / responsive molecularly thin coatings. ${ }^{8}$ Physically adsorbed polymers have been widely used in adhesion and lubrication technology, antifouling and tissue engineering. ${ }^{9}$

To cite only a few example of these technologies we can mention the use of grafted polymer brushes to reduce friction, ${ }^{10}$ to create stimuli responsive thin-film devices ${ }^{11-12}$ or to develop fouling resistant substrates. ${ }^{13}$ Self-adsorbed polymers such as tribloc polymers, have been suggested as adhesive polymers and friction reducing coatings as well. ${ }^{14}$ Large molecular weight polymer are often used to create biomimetic microcellular environments, ${ }^{15}$ a useful strategy in the design of bio - sensors/ detectors ${ }^{16}$ and microfluidic analysis platforms ${ }^{17}$.

Besides the vast number of study demonstrating the versatility of polymers in tuning interfacial properties, these have been mostly been focused on linear polymer chains rather than more complex polymer architectures such as dendritic, comb-like or even ring polymers. A few recent examples using dendritic polymer coatings as antifouling coating have demonstrated that complex architectures can provide superior interfacial properties compared to all linear polymers. $^{17-22}$ 
Complex polymeric architectures are abundantly found in nature, especially in proteins and are known to provide very specific properties to them. For example, proteins found in articular joints exhibiting the bottle brush architecture are known to be excellent lubricants. ${ }^{23}$ Many transmembrane proteins and lipids exhibit dendritic glycosylated moieties at their surface which are known to modulate ligand-receptors interactions. ${ }^{24}$ These few examples suggest that polymer architecture is an important factor that can be used in engineered systems to control polymer conformation and interfacial properties of surfaces.

In the present study, we focused on the interfacial properties of star-shaped polymers bearing adhesive blocks at the extremities of their arms. Star-shaped polymers have demonstrated interesting properties as drug delivery systems ${ }^{25-26}$ and they offer interesting possibilities in hydrogel design ${ }^{27}$. Since their behavior at solid-liquid interfaces is largely unknown, we used the Surface Forces Apparatus to characterize the interaction forces between two apposing surfaces functionalized with such polymers. The polymers were self-adsorbed onto the surfaces, and their conformation was monitored at different surface concentrations. The analysis of the results has allowed to unravel the large variety of conformational changes exhibited by our system and has identified a key role of water in the molecular mechanism involved in the polymer-substrate interaction. 


\section{Materials and method}

Materials. Ruby Mica blocks (Grade I V-1) were purchased from SJ Trading, USA. Pure water was obtained from a Millipore purification system (Synthesis A10) equipped with a Quantum EX purification column. All the polymers used in the present study were synthesized and characterized in a previous report. ${ }^{28}$

Methods. Interaction forces were measured in a Surface Forces Apparatus (SFA 2000, Surforce, LLC). Prior to the study, the apparatus was passivated by immersion in concentrated nitric acid for $24 \mathrm{~h}$ and extensive rinsing with pure water. Surfaces were prepared as previously reported ${ }^{29-}$ ${ }^{30}$. Briefly, two back-silvered mica pieces $(2.5 \mu \mathrm{m}$ thick) were glued (silvered face down) on cylindrical glass disks with thermoset glue Epon ${ }^{\circledR}$ 1004. After positioning the surfaces in a cross configuration in the SFA, the measurement chamber was purged with argon for 15 minutes. After purging, the surfaces were brought to adhesive contact in order to quantify the mica thickness. To do so, a white light beam is directed through the surfaces and the interference fringes (also called fringes of equal chromatic order, FECO) are recorder in a spectrophotometer (Shamrock ${ }^{\circledR} 500$, Andor, USA) equipped with a CMOS camera (Zyla, Andor, USA). Once the FECO analysis is performed ${ }^{31}$, the surfaces are separated again and $25 \mu \mathrm{L}$ of polymer solution is delicately introduced between them. After an equilibration time of $1 \mathrm{~h}$, the surfaces a brought together and force measurements are performed as already reported ${ }^{32}$ using a motor controlled displacement system allowing for the displacement speed to be as low as $0.3 \mathrm{~nm} / \mathrm{s}$.

To change the concentration of polymer in the solution or to rinse the surfaces, the injected solution was carefully sucked out using a $1 \mathrm{~mL}$ tuberculine syringe (BD syringe) and replaced by $25 \mu \mathrm{L}$ of new solution or pure water. This procedure was repeated six times before equilibrating again for one hour. 


\section{Results and discussion}

Polymers' characteristics in solution.

The polymers used in the present study are described in Figure 1. The core of the star-shaped polymer is constituted of cholic acid (CA). In its native form, cholic acid is known to be amphiphilic with a critical micellar concentration around 10 to $16 \mathrm{mM}$ in water at $25^{\circ} \mathrm{C}^{33}$. The facial amphiphily of CA is provided by the preferential orientation of the three hydroxyl groups and the carboxylic function surrounding its cholane core. Cholane precursor with four hydroxyl

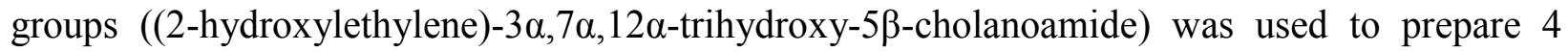
arms block copolymers $\mathrm{CA}\left(\mathrm{EG}_{n}-b-\mathrm{AGE}_{m}\right)_{4}$ by sequence anionic polymerization of ethylene oxide (EG) and allyl glycidyl ether (AGE). Size exclusion chromatography (SEC) confirmed narrow distribution and ${ }^{1} \mathrm{H}$ NMR spectroscopy confirmed the degree of polymerization of both block and the success of the addition of cysteamine by thiolene click chemistry (e.i $\mathrm{CA}\left(\mathrm{EG}_{n}-b-\right.$ $\left.\mathrm{AGE}_{m}-\mathrm{NH}_{2}\right)_{4}$. In the present study, a fourth hydroxyl group was introduced in the structure via the conjugation of ethanol amine to the carboxylic function present in CA creating a fourth hydroxyl initiation site. The anionic polymerization process allows to obtain highly monodisperse precursors $\mathrm{CA}\left(\mathrm{EG}_{\mathrm{n}}-b-\mathrm{AGE}_{\mathrm{m}}\right)_{4}$ with $\mathrm{PDI}<1,11$ (see Table 1$)$.

Table 1. Summary of the physical properties of the polymers used ${ }^{28}$

\begin{tabular}{|c|c|c|c|c|c|c|}
\hline & $\mathrm{Mn}(\mathrm{g} / \mathrm{mol})$ & $\mathrm{Mn}(\mathrm{g} / \mathrm{mol})$ & \multirow{2}{*}{$\begin{array}{c}\text { EG per } \\
\text { arm }\end{array}$} & \multirow{2}{*}{$\begin{array}{l}\text { Amine group } \\
\text { per arm }\end{array}$} & \multirow{2}{*}{$\begin{array}{l}\text { Contour length } \\
\text { of } 1 \mathrm{arm}(\mathrm{nm})\end{array}$} & \multirow[t]{2}{*}{ CAC mM } \\
\hline & SEC (PDI) & $\mathrm{NMR}^{1} \mathrm{H}$ & & & & \\
\hline $\mathrm{CA}\left(\mathrm{EG}_{28}-b-\mathrm{AGE}_{11}\right)_{4}$ & $12,140(1.11)$ & & 28 & & & \\
\hline $\mathrm{CA}\left(\mathrm{EG}_{28}-b-\mathrm{AGE}_{11}-\mathrm{NH}_{2}\right)_{4}$ & & 13500 & 28 & 11 & 10.2 & 0.82 \\
\hline $\mathrm{CA}\left(\mathrm{EG}_{21}-b-\mathrm{AGE}_{11}\right)_{4}$ & $10,100(1.06)$ & & 21 & & & \\
\hline $\mathrm{CA}\left(\mathrm{EG}_{21}-b-\mathrm{AGE}_{11}-\mathrm{NH}_{2}\right)_{4}$ & & 12900 & 21 & 11 & 7.6 & 0.47 \\
\hline $\mathrm{CA}\left(\mathrm{EG}_{11}-b-\mathrm{AGE}_{11}\right)_{4}$ & $8680(1.07)$ & & 11 & & & \\
\hline $\mathrm{CA}\left(\mathrm{EG}_{11}-b-\mathrm{AGE}_{11}-\mathrm{NH}_{2}\right)_{4}$ & & 11800 & 11 & 11 & 4.0 & 0.40 \\
\hline
\end{tabular}

Post-functionalisation of the allyl functional groups lead to eleven terminal amino groups on each branch. In a previous report ${ }^{28}$, we showed that the amine functions have a pKa $=8.5( \pm 0.2)$ 
for all the $\mathrm{CA}\left(\mathrm{EG}_{n}-b-\mathrm{AGE}_{m}-\mathrm{NH}_{2}\right)_{4}$ in water. Therefore, we expect these functional groups to be fully protonated at $\mathrm{pH}$ below 7.5 at room temperature. Three polymers, with different numbers of EG units per arm were chosen and studied using the Surface Forces Apparatus (SFA).
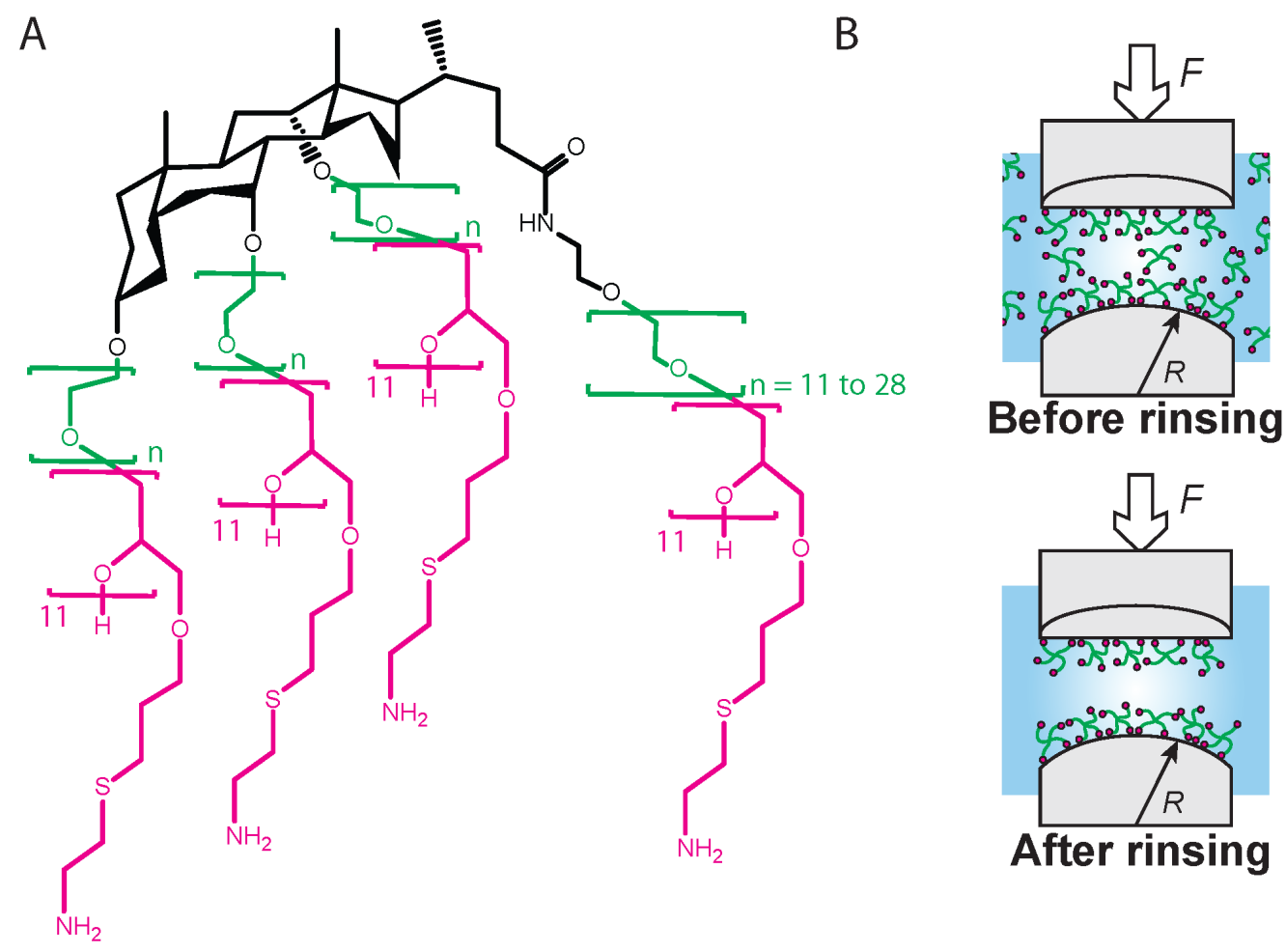

After rinsing

Figure 1. A) Generic chemical structure of the different polymers used in the present study. B) Schematic representation of the two different experimental configurations used during the measurements of the interaction forces across polymer solutions. The upper configuration corresponds to the surfaces interacting across a solution of free and adsorbed polymer solution while in the lower configuration, the surfaces were extensively rinsed with pure water to leave only adsorbed polymer on the surfaces.

\section{General features of the interaction forces measured across solutions of star-shaped polymers}

We performed a series of interaction force measurements between mica surfaces in presence of the different polymers at three concentrations, $C_{\mathrm{p}}$. The interaction force $F$ was normalized by the surface radius so the data could be interpreted in terms of interaction energy, $W$, via the Derjaguin approximation $(F=2 \pi R W, W$ being the interaction energy between two flat 
surfaces). ${ }^{34}$ At each $C_{\mathrm{p}}$, the interaction forces were measured first across the polymer solution and a second time across pure water (after removing the polymer solution and rinsing abundantly the surfaces with pure water) at the same contact (see figure 1B). In Fig. 2, we show the different force laws, also called force profiles $(F / R v s$ separation distance $D)$ before and after rinsing the surfaces, obtained on approach only. For all three polymers, at $C_{\mathrm{p}}=2 \mu \mathrm{g} / \mathrm{mL}$, the force profiles exhibited two different regimes: a long range, exponentially decaying interaction regime, and a shorter range, sharply decaying regime presenting occasional instabilities. Such behavior was observed systematically under rinsed conditions and happened only for the highest molecular weight polymer in non-rinsed conditions. The short-range part of the force profiles was characterized by a steep increase of the interaction forces until an abrupt "jump-in" transition occurred (indicated as "squeeze out" in Figure 2). As will be explained later, this first jump-in transition can be ascribed to a conformational change of the confined polymer chains. Further compression of the surfaces led to the occurrence of other similar transitions of smaller magnitude, which will be discussed below.

An increase in $C_{\mathrm{p}}$ led to a systematic increase of the onset of the interaction forces indicating that the forces were largely dominated by the compression of the polymer layers (Figure 2). Interaction forces measured after rinsing the surfaces were systematically weaker compared to those measured across the corresponding polymer solution, indicating that weakly adsorbed polymer chains have been washed off the surfaces during the rinsing process.

It is interesting to note that at $C_{\mathrm{p}}>2 \mu \mathrm{g} / \mathrm{mL}$, the interaction force profile exhibited a single exponential decay for the low molecular weight polymer and evolved towards a two exponential 
decay for the higher molecular weight polymers. We will see that these gradual changes in the force law are reminiscent of a change in the inner structure of the adsorbed polymer film.
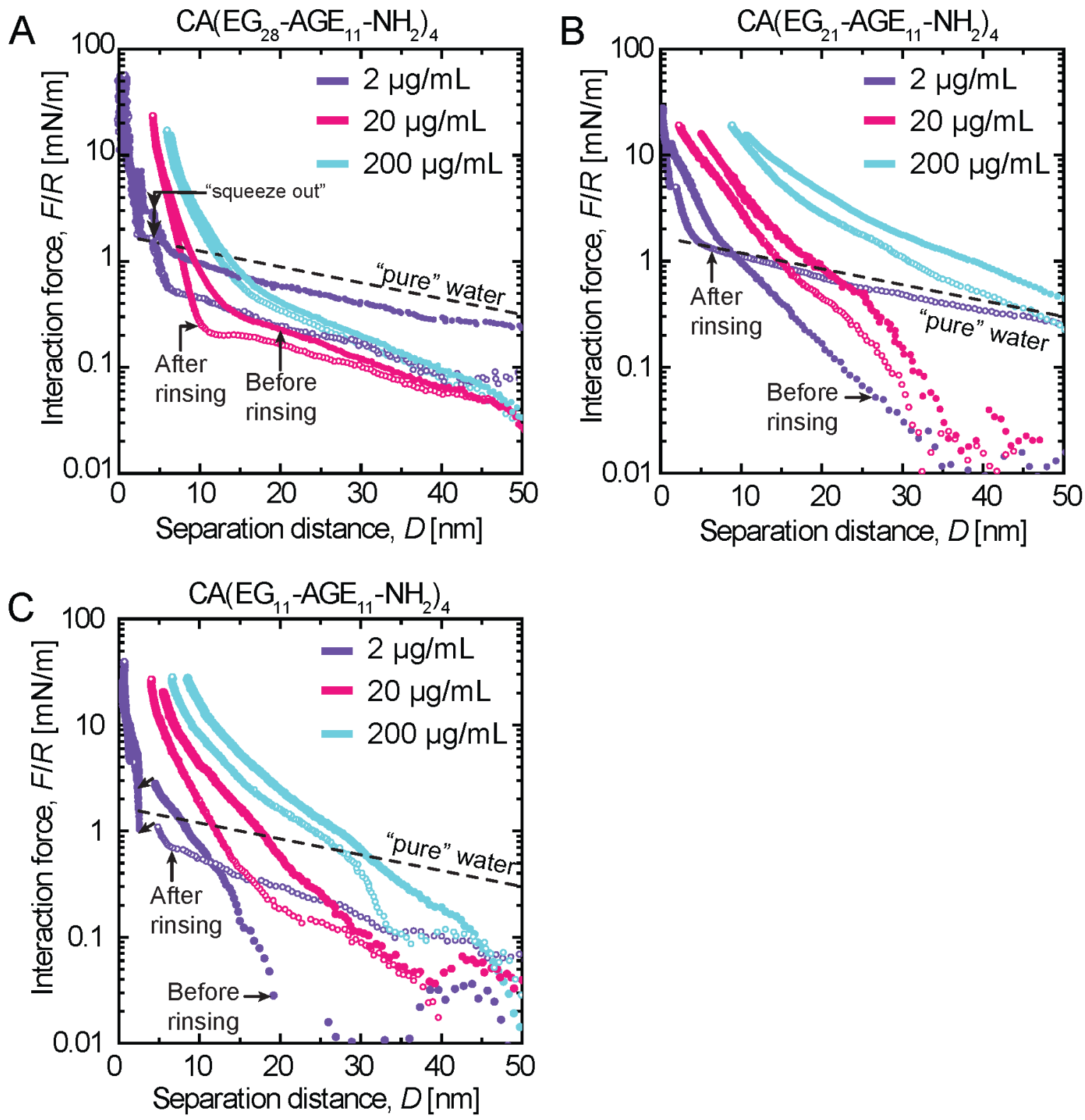

Figure 2. A-C) Interaction forces measured across solutions of a star-shaped polymer (filled symbols) and across water after rinsing the surfaces (open symbols) for all three polymers tested. The long-range portion of the force profile corresponds to the overlapping of the electrostatic double layers, while the short range portion is indicative of the compression of the polymer and the hydration layers. 


\section{Analysis of the electrosteric forces under rinsed conditions}

Given the complexity of the polymers' architecture and the large number of possible conformations these can adopt at the surfaces, certain assumptions had to be made in order to analyse the force profiles shown in Figures 2 . In what follows, we analyzed the experimental data obtained after rinsing only.

In pure water, the amine groups positioned at the end of each branch were expected to be positively charged (at least in the range of $C_{\mathrm{p}}$ this study was performed) and therefore to interact strongly with the negatively charged mica surface. At low surface coverage (low $C_{\mathrm{p}}$ ), the polymer was expected to interact with the surface via his four branches forming a loop. Some chains will certainly have only three or two branches adsorbed at the surface leaving the other one or two dangling in the medium. As $C_{\mathrm{p}}$ increases, adsorption sites were gradually becoming scarcer leaving the polymer chains to interact with the substrate via fewer branches. At high surface coverage, adsorbing chains would be expected to interact with the surface via one branch only adopting a "flower-like" conformation. This last conformation has been extensively studied experimentally $^{19,35-36}$ and especially theoretically ${ }^{37-39}$. The so-called dendronized end- grafted brushes have been shown to provide significantly more stability to colloidal particles compared to linear brushes ${ }^{36}$ and have also demonstrated far superior anti-fouling capacity compared to their linear equivalent ${ }^{19}$. Theoretical models describing the conformation and interactions of dendronized brushes have matured during the past few years ${ }^{40}$. As for their linear equivalent, these models use the scaling law approach to derive the free energy per macromolecule at the surface. In this framework, the free energy of the brush, $E$, is the sum of the repulsive excluded volume interaction between monomer units forming the brush and the conformational entropy of the brush. The interaction forces measured with the SFA are related to the interaction energy 
between the surfaces, $W$, and to the brush free energy $E$ via the Derjaguin approximation which $\operatorname{reads}^{34}$ :

$$
\frac{F(D)}{R}=2 \pi W(D)=2 \pi \Gamma[E(D)-E(H)]
$$

where $\Gamma$ is the polymer surface coverage. An expression of the interaction energy between surfaces bearing dendronized brushes was proposed by Borisov ${ }^{38}$ :

$$
\frac{F(D)}{R}=\frac{10 \pi}{9} \Gamma E(H)\left[\left(\frac{D}{2 H}\right)^{2}-\frac{1}{5}\left(\frac{D}{2 H}\right)^{5}+\frac{2 H}{D}\right]
$$

with $\frac{E(H)}{k_{B} T}=\frac{9}{20} \frac{k^{4}}{v a^{7}} H^{5}$

where $H$ is the brush thickness, $a$ is the segment length, $v$ is the dimensionless second virial coefficient and $k \approx n^{-1} q^{-1 / 2}$ (n being the branch length and $q$ the number of non adsorbed branches).

At low $C_{\mathrm{p}}$, since the force profiles presented a two exponential decay, we used Eq. 2 to analyze the short range part of the force profiles and the Derjaguin Landau Verwey Overbeeck (DLVO) theory for the long range part. Indeed, we noticed that the characteristic decay length of the long range forces was close to the value measured in pure water (Table 2), therefore we concluded that this portion of the force profile was due to electrostatic interactions only. The interaction force law can be written as ${ }^{41}$ :

$$
W=W_{E S}+W_{v d W}
$$

where 


$$
\begin{aligned}
& W_{v d W}=-\frac{A_{H}}{6 D^{2}} \\
& C P: W_{E S}=2 \varepsilon_{0} \varepsilon_{r} \frac{\psi_{0}^{2}}{\lambda} \ln \left[1+e^{-D / \lambda}\right] \\
& C C: W_{E S}=-2 \varepsilon_{0} \varepsilon_{r} \frac{\psi_{0}^{2}}{\lambda} \ln \left[1-e^{-D / \lambda}\right]
\end{aligned}
$$

where $W_{\mathrm{ES}}$ and $W_{\mathrm{vdW}}$ are the electrostatic and van der Walls contributions to the total interaction energy, $A_{\mathrm{H}}$ is the Hamaker constant (equal to $2.2 \times 10^{-20} \mathrm{~J}$ for mica in water), $\psi_{0}$ is the surface potential and $\lambda$ is the Debye length. Equation (5) and (6) are derived using the constant potential (CP) or constant charge (CC) boundary condition. Previous experiments have shown that interaction forces between mica surfaces in pure water follow more closely the CC condition ${ }^{34}$, 42 at short separation distances. Our experiments described a similar behaviour at low $C_{\mathrm{p}}$ (see Figure 3A).

At higher $C_{\mathrm{p}}$, when only a single exponential decay was observed, Eq. 2 was used to fit the force runs. In figure $3 \mathrm{~A}$, we showed a typical fitting for the polymer $\mathrm{CA}\left(\mathrm{EG}_{28}-b-\mathrm{AGE}_{11} \mathrm{NH}_{2}\right)_{4}$ at $C_{\mathrm{p}}=$ $2 \mu \mathrm{g} / \mathrm{mL}$.

The fitted values of the surface potential and Debye length are given in Table 2. As a reference, we measured the interaction forces between two mica surfaces in pure water only. The obtained Debye length was $\lambda \sim 55 \mathrm{~nm}$, which was significantly smaller than the expected theoretical value for pure water (around $960 \mathrm{~nm}$ ). Purified water has a $\mathrm{pH}=5.8$, corresponding to an equivalent concentration of 1:1 electrolyte of $3.6 \times 10^{-6} \mathrm{M}$ which gives a decay length of $160 \mathrm{~nm}$. The effective ion concentration in pure water calculated using $\lambda \sim 55 \mathrm{~nm}$ was $3.0 \times 10^{-5} \mathrm{M}$, which was well above the predicted value. The main reason for this difference is the presence of 
background electrolytes that were introduced by the polymer itself besides extensive dialysis. At low $C_{\mathrm{p}}$, the long-range decay length in presence of polymer chains at the surfaces only (after rinsing) was slightly smaller than pure water as shown in Table 2 . This could be explained by small variations in electrolyte concentration in the medium (due to the presence of small amount of free polymer for example).

Table 2. Fitted parameters obtained using equations Eq. 2-6

\begin{tabular}{cccccc}
$\begin{array}{c}\text { EG units } \\
\text { per } \\
\text { branch }\end{array}$ & $\begin{array}{c}C_{\mathrm{p}} \\
{[\mu \mathrm{g} / \mathrm{ml}]}\end{array}$ & $\begin{array}{c}\text { Debye length } \\
\lambda[\mathrm{nm}]\end{array}$ & $\begin{array}{c}\text { Surface potential } \\
\psi_{0}[\mathrm{mV}]\end{array}$ & $\begin{array}{c}\text { Brush heigt } H \\
{[\mathrm{~nm}]}\end{array}$ & $\begin{array}{c}\text { Number of non } \\
\text { adsorbed branches, } q\end{array}$ \\
\hline \multirow{5}{*}{11} & 0 & 55 & -172 & - & - \\
& 2 & 21.5 & -95 & 5.2 & 1 \\
& 20 & 5 & -73 & 15 & 3 \\
& 200 & - & - & 29.5 & 3 \\
21 & 20 & 41 & -147 & 4.8 & 1 \\
& 20 & 25 & -43 & 15 & 2 \\
& 2 & - & - & 40 & 3 \\
28 & 20 & 25 & -85 & 5 & 1 \\
& 20 & 14 & -70 & 5 & 3 \\
\hline
\end{tabular}

\section{Analysis of the steric contribution}

In Table 2 we summarized the results of the fittings using Eq. 2 for the most important parameters, namely the number of non-adsorbed branches $q$, and the brush height $H$. We clearly see that as $C_{\mathrm{p}}$ increases from 2 to $200 \mu \mathrm{g} / \mathrm{mL}$, the dendronized brush model predicts that the number of non-adsorbed branches as well as the brush height increase, independently of the molecular weight of the branches (Fig. 3B). At $C_{\mathrm{p}}=200 \mu \mathrm{g} / \mathrm{mL}$, the values obtained for the brush height $H$ were significantly larger than the size of any of the tested polymers (see Table 2) suggesting that the polymer layer structure was composed of more than one monolayer besides the extensive rinsing. The adsorbed polymer film was most likely constituted of polymer 
aggregates strongly adsorbed on the mica substrate (see schematic in Fig. 3B). The cohesive interactions mediating the aggregation of the polymers involved the hydrophobic attraction between the CA cores of the polymers.
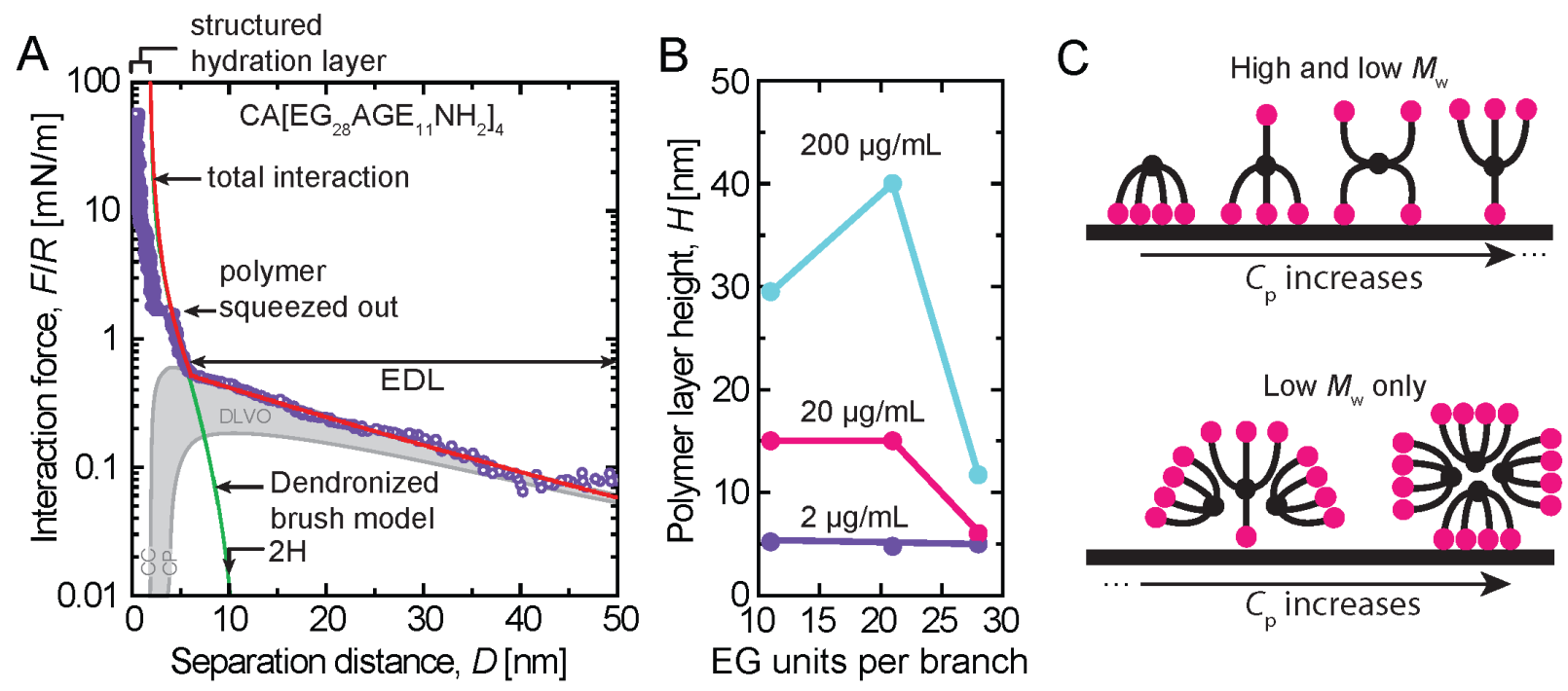

Figure 3. A) Interfacial structuring of the adsorbed polymer layer on mica. Interaction forces (measured at $2 \mu \mathrm{g} / \mathrm{mL}$ ) confirmed the presence of an electrostatic double layer (EDL) superimposed with a polymer layer. A third layer of structured hydration water is also evident below $D=3 \mathrm{~nm}$. B) The evolution of the polymer layer thickness with the number of EG units per branch of polymer shows a strong increase with concentration, especially for the smallest polymers. As illustrated in C), such increase in layer thickness indicates the aggregation of the smallest polymers at the surface.

It is interesting to note that the onset of the interaction forces measured at $C_{\mathrm{p}}=200 \mu \mathrm{g} / \mathrm{mL}$, which is equal to $2 H$, is smaller for large $M_{\mathrm{w}}$ branches compared to smaller branches (see Figure 3B). Such trend suggests that the size of the polymer aggregates might be larger for the low molecular weight macromolecules. Previous report ${ }^{28}$ have shown that the critical aggregation concentration, $C A C$, of the polymers increases as the molecular weight of the polymer increases (see Table 1). Such observation suggests that the large increment in the onset of interaction observed for the smallest polymers is due to the formation of large micellar aggregates. It is important to note that the values of the $C A C$ reported in Table 1 were obtained in solution by 
viscosimetry analyses and were significantly higher than the concentrations of the tested solutions in the SFA. The aggregation of the star-shaped polymer observed in the SFA experiments was therefore induced by the first layer of polymer adsorbed on the surfaces which acted as a nucleation site for the aggregation process.

\section{Analysis of the hydration forces}

As we have seen, at high $C_{\mathrm{p}}$, the measured interaction forces were largely dominated by the compression of the polymer layers adsorbed on the substrates. On the other hand, at low $C_{\mathrm{p}}(=2$ $\mu \mathrm{g} / \mathrm{mL}$ ), we noticed that for all the tested polymers, the interaction forces also presented regular instabilities appearing as sudden variations in $D$. More precisely, at $D=2.5-4.5 \mathrm{~nm}$, a first abrupt jump of $D$ at almost constant force $F / R=1-2 \mathrm{mN} / \mathrm{m}$ was systematically observed (Figure 2 and 3A). Such an instability is indicative of a sudden squeeze out of the polymer from the contact leaving the interaction area empty of polymer chains. The size of this sudden distance jump was $\Delta D=1.5-2.5 \mathrm{~nm}$, which is close to the expected theoretical value of the radius of gyration $R \mathrm{~g}$ of the polymer $\left(\sim 2.7-3.8 \mathrm{~nm} \text { depending on the branch } M_{\mathrm{w}}\right)^{43}$. As shown in Figure 2B, interaction forces measured before the squeeze out of the polymer layers $(D>5 \mathrm{~nm})$ were systematically found to be repulsive $(F / R>0)$ and reversible. After the squeeze out of the polymer layer (which occurred only at $C_{\mathrm{p}}=2 \mu \mathrm{g} / \mathrm{mL}$ for the range of applied forces used in this study), the interaction forces measured were not reversible anymore. Instead, strong adhesion minima were found to appear upon separation of the surfaces whose magnitude was strongly dependent upon the minimum separation distance / highest force applied (see Fig. S1). Such drastic change in the behavior of the interaction forces was accompanied by the appearance of small instabilities, similar to those observed during the squeeze out of the polymer except in smaller magnitude $(\Delta D=0.25 \mathrm{~nm})$. Since no polymer chains were present in the contact, this 
force regime corresponds to the compression of the mica hydration layers. As shown in Figure $4 \mathrm{~A}$ and $\mathrm{B}$, the length of the polymers' branches did not modify significantly the appearance of this regime.

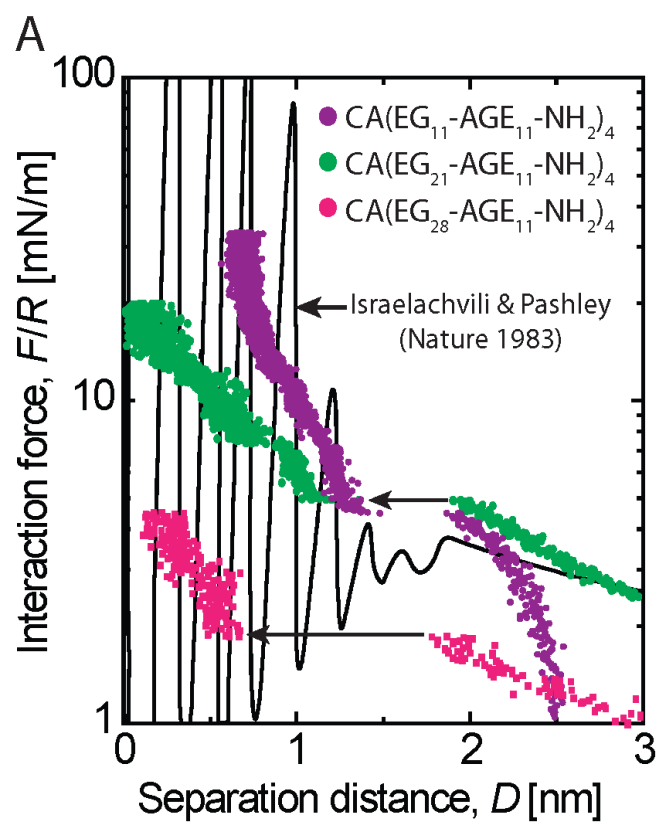

B
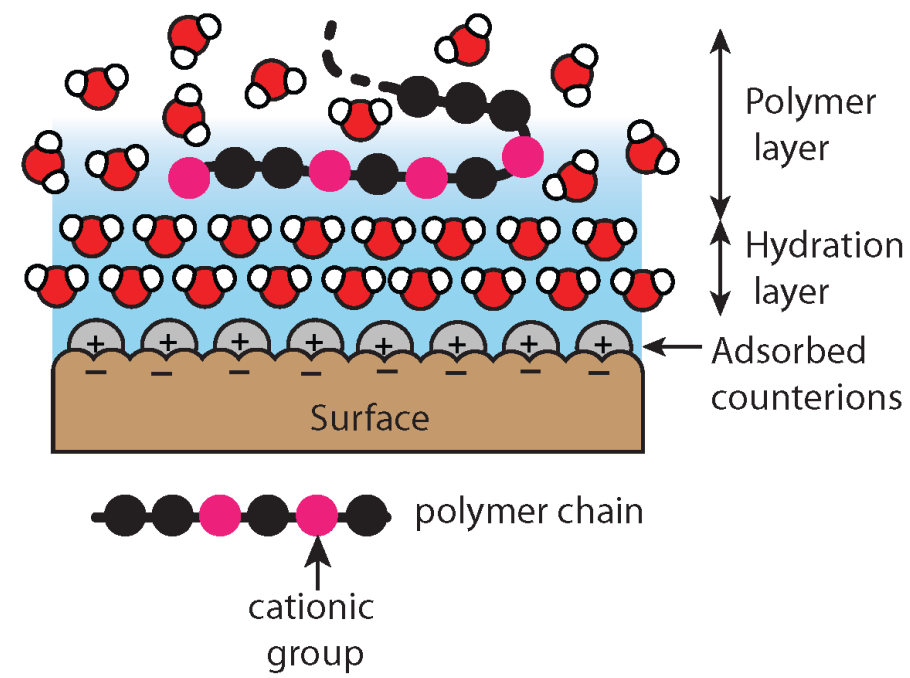

Figure 4. A) Interaction forces measured between $0-3 \mathrm{~nm}$ in presence of the star-shaped polymers $(2 \mu \mathrm{g} / \mathrm{mL})$. The step-like form of the force profile matches very closely the oscillatory force profile observed for pure hydration forces represented by the black line. B) Schematic representation of the solid-liquid interface with an adsorbed polymer chain. The positively charged extremity of the polymer branches seats on multiple layers of water molecules strongly anchored to the substrate.

Superposition of the force profiles measured after rinsing the surfaces (e.g range of 0 to $3 \mathrm{~nm}$ ), with a previous report on hydration forces between mica surface, suggested similarities in the position of the instabilities measurement and the oscillatory profile obtained with water by Israelachvili and Pashley ${ }^{42}$ (Figure 4A). The figure shows remarkable similarities in the position of the instabilities measured in our system and the oscillatory force profiles obtained with water. These similarities suggest that the observed instabilities are due to the layering of water molecules at the mica surfaces, phenomena that has been shown to be induced by the presence of positively charged ions adsorbed at the surfaces ${ }^{44}$. As illustrated in figure $4 \mathrm{~B}$, our results imply 
that the adsorbed polymer chains were not in direct molecular contact with the substrate but rather interacted indirectly with it through the highly ordered layers of immobilized water molecules.

These results echoes similar observations we recently reported with hyaluronic acid adsorption on mica surfaces ${ }^{29}$. HA is a negatively charged polyelectrolyte, which adsorbs on mica in a relatively short time when its molecular weight $M_{\mathrm{w}}$ is about $10 \mathrm{kDa}$. Given that the polymer is negatively charged in water, same as mica, the attraction between the polymer to the surface was expected to be mediated by hydrogen bonding. At low ionic strength, HA was found to adsorb strongly on the surfaces while its adsorption was dramatically hampered by increasing the salt concentration and even totally eliminated at a salt concentration of $1.5 \mathrm{M}$. The decrease of HA adsorption was correlated with the appearance of a 2-3 nm thick hydration layer composed of multiple layers of water molecules at the solid-liquid interface.

The role of surface hydration layers has been overlooked in adsorption studies of polymers and especially for polyelectrolytes. In the case of oppositely charged polyelectrolytes and surfaces, it is well established that the driving force leading to adsorption is the electrostatic attraction between the surface adsorption site and the ionized monomer. ${ }^{45-47}$ The ionic strength of the medium has been shown to screen this interaction and therefore to decrease the amount of adsorbed polymer and concomitantly to increase the thickness of the adsorbed layer. ${ }^{47-48}$ The energy of interaction between a positively charged monomer belonging to an adsorbed chain and a charged surface can be calculated as $E \approx N_{\mathrm{A}} \mathrm{e}^{2} \sigma d /\left(2 \varepsilon_{0} \varepsilon_{\mathrm{r}}\right)$ where $N_{\mathrm{A}}$ is the Avogadro number, e the electron charge, $\sigma$ the surface charge density, $d$ the average distance between adsorbed chains (1-10 nm), and $\varepsilon_{0}$ and $\varepsilon$ the dielectric constant of the vacuum and hydration layer respectively. ${ }^{49}$ For the calculation, the surface charge density was estimated considering the Graham 
approximation using an average surface potential of $100 \mathrm{mV}$ and a dielectric constant $\varepsilon_{\mathrm{r}}=6$ (corresponding to strongly adsorbed water molecules). In this framework, the interaction energy of a single charged monomer with the surface was $\sim-1$ to $-20 \mathrm{kT}$. Close molecular contact of the monomer with the surface also implied partial dehydration of the monomer which, in the case of protonated amines groups represents a $\sim 25 \mathrm{kT}$ energy penalty per water molecule. In addition, the Born energy transfer of a monomer from bulk water to a hydrated, structured layer of water represented a $\sim 30 \mathrm{kT}$ additional energetic penalty. This back of the envelope calculation demonstrated that penetration of a monomer into the hydration layer was overall not energetically favorable even though experimental measurements of the interaction energy of charged amine groups to mica were still missing to refine this analysis. The electrostatic interaction energy of an amine group adsorbed on top of a hydration layer is much weaker but is

compensated by hydrogen bonds between the amine and immobilized water molecules $(\sim 4-10$ kT per bond).

\section{CONCLUSION}

The present study confirms that polymer adsorption to hydrated surfaces can be mediated by layers of strongly adsorbed water molecules. We can easily anticipate that the structure of such layers is critical in determining the stability and strength of the polymer chain adsorption and conformation. These results also suggested that polymer conformation and adsorption to a surface can be finely engineered via the structuring of the surface hydration layer. Recent reports have demonstrated that certain combinations of functional groups are significantly more efficient to locally break the surface hydration layer facilitating the close contact of molecules with the 
surface's atoms. ${ }^{50}$ Our observations suggested that a similar approach could be used to design polymers exhibiting chaotropic or cosmotropic properties towards hydrated surfaces.

\section{AUTHOR INFORMATION}

\section{Corresponding Author}

xavier.banquy@umontreal.ca.

\section{Present Addresses}

$\dagger$ Leslie Dan Faculty of Pharmacy, Department of Pharmaceutical Sciences, University of Toronto, Canada.

\section{Author Contributions}

The manuscript was written through contributions of all authors. All authors have given approval to the final version of the manuscript.

\section{Funding Sources}

\section{ACKNOWLEDGMENT}

XB is grateful for the financial support of the Canada Research Chair program and to NSERC (Discovery grant).

\section{Supporting information}

Force profiles performed at different maximum applied force. 


\section{REFERENCES}

1. $\quad$ Polymer Thin Film. World Scientific: Hackensack, NJ USA, 2008; Vol. 1.

2. $\quad$ Crevoisier, G. B.; Fabre, P.; Corpart, J. M.; Leibler, L. Switchable Tackiness and Wettability of a Liquid Crystalline Polymer Science 1999, 285, 1246-1249.

3. $\quad$ Feng, C. L.; Zhang, Y. J.; Jin, J.; Song, Y. L.; Xie, L. Y.; Qu, G. R.; Jiang, L.; Zhu, D. B. Reversible Wettability of Photoresponsive Fluorine-Containing Azobenzene Polymer in Langmuir-Blodgett Films Langmuir 2001, 17, 4593-4597.

4. Klein, J. Shear, Friction, and Lubrication Forces between Polymer-Bearing Surfaces Annu Rev Mater Sci 1996, 26, 581-612.

5. $\quad$ Leger, L.; Raphael, E.; Hervet, H. Surface-Anchored Polymer Chains: Their Role in Adhesion and Friction Adv Polym Sci 1999, 138, 185-225.

6. Nie, Z. H.; Kumacheva, E. Patterning Surfaces with Functional Polymers Nat Mater 2008, 7, 277-290.

7. Zhao, B.; Brittain, W. J. Polymer Brushes: Surface-Immobilized Macromolecules Prog Polym Sci 2000, 25, 677-710.

8. Zhai, L. Stimuli-Responsive Polymer Films Chem Soc Rev 2013, 42, 7148-7160.

9. Krishnan, S.; Weinman, C. J.; Ober, C. K. Advances in Polymers for Anti-Biofouling Surfaces J Mater Chem 2008, 18, 3405-3413.

10. Raviv, U.; Giasson, S.; Kampf, N.; Gohy, J. F.; Jerome, R.; Klein, J. Lubrication by Charged Polymers Nature 2003, 425, 163-165.

11. Tokarev, I.; Minko, S. Stimuli-Responsive Hydrogel Thin Films Soft matter 2009, 5, 511 524.

12. Pandeeswar, M.; Senanayak, S. P.; Narayan, K. S.; Govindaraju, T. Multi-StimuliResponsive Charge-Transfer Hydrogel for Room-Temperature Organic Ferroelectric Thin-Film Devices J Am Chem Soc 2016, 138, 8259-8268.

13. Banerjee, I.; Pangule, R. C.; Kane, R. S. Antifouling Coatings: Recent Developments in the Design of Surfaces That Prevent Fouling by Proteins, Bacteria, and Marine Organisms $A d v$ Mater 2011, 23, 690-718.

14. Kang, T.; Banquy, X.; Heo, J.; Lim, C.; Lynd, N. A.; Lundberg, P.; Oh, D. X.; Lee, H. K.; Hong, Y. K.; Hwang, D. S.; Waite, J. H.; Israelachvili, J. N.; Hawker, C. J. Mussel-Inspired Anchoring of Polymer Loops That Provide Superior Surface Lubrication and Antifouling Properties ACS Nano 2016, 10, 930-937.

15. Hanauer, N.; Latreille, P. L.; Alsharif, S.; Banquy, X. 2d, 3d and 4d Active Compound Delivery in Tissue Engineering and Regenerative Medicine Curr Pharm Des 2015, 21, 15061516.

16. Wang, X. Y.; Kim, Y. G.; Drew, C.; Ku, B. C.; Kumar, J.; Samuelson, L. A. Electrostatic Assembly of Conjugated Polymer Thin Layers on Electrospun Nanofibrous Membranes for Biosensors Nano Lett 2004, 4, 331-334.

17. Huber, D. L.; Manginell, R. P.; Samara, M. A.; Kim, B. I.; Bunker, B. C. Programmed Adsorption and Release of Proteins in a Microfluidic Device Science 2003, 301, 352-354.

18. Weinhart, M.; Becherer, T.; Schnurbusch, N.; Schwibbert, K.; Kunte, H. J.; Haag, R. Linear and Hyperbranched Polyglycerol Derivatives as Excellent Bioinert Glass Coating Materials Adv Eng Mater 2011, 13, B501-B510.

19. Gillich, T.; Benetti, E. M.; Rakhmatullina, E.; Konradi, R.; Li, W.; Zhang, A.; Schluter, A. D.; Textor, M. Self-Assembly of Focal Point Oligo-Catechol Ethylene Glycol Dendrons on 
Titanium Oxide Surfaces: Adsorption Kinetics, Surface Characterization, and Nonfouling Properties J Am Chem Soc 2011, 133, 10940-10950.

20. Wei, Q.; Achazi, K.; Liebe, H.; Schulz, A.; Noeske, P. L. M.; Grunwald, I.; Haag, R. Mussel-Inspired Dendritic Polymers as Universal Multifunctional Coatings Angew Chem, Int Ed Engl 2014, 53, 11650-11655.

21. Wei, Q; Becherer, T.; Noeske, P. L. M.; Grunwald, I.; Haag, R. A Universal Approach to Cross Linked Hierarchical Polymer Multi Layers as Stable and Highly Effective Antifouling Coatings Adv Mater 2014, 26, 2688-2693.

22. Wei, Q.; Haag, R. Universal Polymer Coatings and Their Representative Biomedical Applications Mater Horiz 2015, 2, 567-577.

23. Swann, D. A.; Silver, F. H.; Slayter, H. S.; Stafford, W.; Shore, E. The MolecularStructure and Lubricating Activity of Lubricin Isolated from Bovine and Human Synovial-Fluids Biochem J 1985, 225, 195-201.

24. Moremen, K. W.; Tiemeyer, M.; Nairn, A. V. Vertebrate Protein Glycosylation:

Diversity, Synthesis and Function Nat Rev Mol Cell Biol 2012, 13, 448-462.

25. Le Devedec, F.; Fuentealba, D.; Strandman, S.; Bohne, C.; Zhu, X. X. Aggregation Behavior of Pegylated Bile Acid Derivatives Langmuir 2012, 28, 13431-13440.

26. Le Devedec, F.; Strandman, S.; Hildgen, P.; Leclair, G.; Zhu, X. X. Pegylated Bile Acids for Use in Drug Delivery Systems: Enhanced Solubility and Bioavailability of Itraconazole $\mathrm{Mol}$ Pharmaceutics 2013, 10, 3057-3066.

27. Danmark, S.; Aronsson, C.; Aili, D. Tailoring Supramolecular Peptide-Poly(Ethylene Glycol) Hydrogels by Coiled Coil Self-Assembly and Self-Sorting Biomacromolecules 2016, 17, 2260-2267.

28. Le Devedec, F.; Strandman, S.; Baille, W. E.; Zhu, X. X. Functional Star Block Copolymers with a Cholane Core: Thermo-Responsiveness and Aggregation Behavior Polymer 2013, 54, 3898-3903.

29. Faivre, J.; Shrestha, B. S.; Burdynska, J.; Xie, G.; Moldovan, F.; Delair, T.; Benayoun, S.; David, L.; Matyjaszewski, K.; Banquy, X. Wear Protection without Surface Modification Using a Synergistic Mixture of Molecular Brushes and Linear Polymers ACS Nano 2017, 11, 1762-1769.

30. Banquy, X.; Lee, D. W.; Das, S.; Hogan, J.; Israelachvili, J. N. Shear-Induced Aggregation of Mammalian Synovial Fluid Components under Boundary Lubrication Conditions Adv Funct Mater 2014, 24, 3152-3161.

31. Israelachvili, J. Thin-Film Studies Using Multiple-Beam Interferometry $J$ Colloid Interface Sci 1973, 44, 259-272.

32. Banquy, X.; Burdynska, J.; Lee, D. W.; Matyjaszewski, K.; Israelachvili, J. Bioinspired Bottle-Brush Polymer Exhibits Low Friction and Amontons-Like Behavior J Am Chem Soc 2014, 136, 6199-6202.

33. Paula, S.; Sus, W.; Tuchtenhagen, J.; Blume, A. Thermodynamics of Micelle Formation as a Function of Temperature - a High-Sensitivity Titration Calorimetry Study $J$ Phys Chem 1995, 99, 11742-11751.

34. Israelachvili, J. N., Intermolecular and Surface Forces. 3rd ed.; Academic Press: Burlington, Mass., 2011; 674 p.

35. Walter, A.; Garofalo, A.; Parat, A.; Jouhannaud, J.; Pourroy, G.; Voirin, E.; Laurent, S.; Bonazza, P.; Taleb, J.; Billotey, C.; Elst, L. V.; Muller, R. N.; Begin-Colin, S.; Felder-Flesch, D. 
Validation of a Dendron Concept to Tune Colloidal Stability, Mri Relaxivity and Bioelimination of Functional Nanoparticles J Mater Chem B 2015, 3, 1484-1494.

36. Gillich, T.; Acikgoz, C.; Isa, L.; Schluter, A. D.; Spencer, N. D.; Textor, M. PegStabilized Core-Shell Nanoparticles: Impact of Linear Versus Dendritic Polymer Shell Architecture on Colloidal Properties and the Reversibility of Temperature-Induced Aggregation ACS Nano 2013, 7, 316-329.

37. Borisov, O. V.; Zhulina, E. B. Brushes of Dendritically Branched Polyelectrolytes Macromolecules 2015, 48, 1499-1508.

38. Borisov, O. V.; Zhulina, E. B.; Polotsky, A. A.; Leermakers, F. A. M.; Birshtein, T. M. Interactions between Brushes of Root-Tethered Dendrons Macromolecules 2014, 47, 6932-6945. 39. Lebedeva, I. O.; Zhulina, E. B.; Leermakers, F. A.; Borisov, O. V. Dendron and Hyperbranched Polymer Brushes in Good and Poor Solvents Langmuir 2017, 33, 1315-1325. 40. Leermakers, F. A.; Zhulina, E. B.; Borisov, O. V. Interaction Forces and Lubrication of Dendronized Surfaces Curr Opin Colloid Interface Sci 2017, 27, 50-56.

41. Takamura, K.; Goldsmith, H. L.; Mason, S. G. The Microrheology of Colloidal Dispersions .12. Trajectories of Orthokinetic Pair-Collisions of Latex Spheres in a Simple Electrolyte $J$ Colloid Interface Sci 1981, 82, 175-189.

42. Israelachvili, J. N.; Pashley, R. M. Molecular Layering of Water at Surfaces and Origin of Repulsive Hydration Forces Nature 1983, 306, 249-250.

43. Daoud, M.; Cotton, J. P. Star Shaped Polymers - a Model for the Conformation and Its Concentration-Dependence $J$ Phys(Paris) 1982, 43, 531-538.

44. Ricci, M.; Spijker, P.; Voitchovsky, K. Water-Induced Correlation between Single Ions Imaged at the Solid-Liquid Interface Nature Comm 2014, 5, 4400.

45. Claesson, P. M.; Poptoshev, E.; Blomberg, E.; Dedinaite, A. Polyelectrolyte-Mediated Surface Interactions Adv Colloid Interface Sci 2005, 114, 173-187.

46. Joanny, J. F. Polyelectrolyte Adsorption and Charge Inversion Eur Phys J B 1999, 9 , 117-122.

47. Rojas, O. J.; Claesson, P. M.; Muller, D.; Neuman, R. D. The Effect of Salt

Concentration on Adsorption of Low-Charge-Density Polyelectrolytes and Interactions between Polyelectrolyte-Coated Surfaces J Colloid Interface Sci 1998, 205, 77-88.

48. $\quad$ Dahlgren, M. A. G.; Hollenberg, H. C. M.; Claesson, P. M. The Order of Adding Polyelectrolyte and Salt Affects Surface Forces and Layer Structures Langmuir 1995, 11, 44804485.

49. Dobrynin, A. V.; Deshkovski, A.; Rubinstein, M. Adsorption of Polyelectrolytes at Oppositely Charged Surfaces Macromolecules 2001, 34, 3421-3436.

50. $\quad$ Maier, G. P.; Rapp, M. V.; Waite, J. H.; Israelachvili, J. N.; Butler, A. Adaptive Synergy between Catechol and Lysine Promotes Wet Adhesion by Surface Salt Displacement Science 2015, 349, 628-632. 


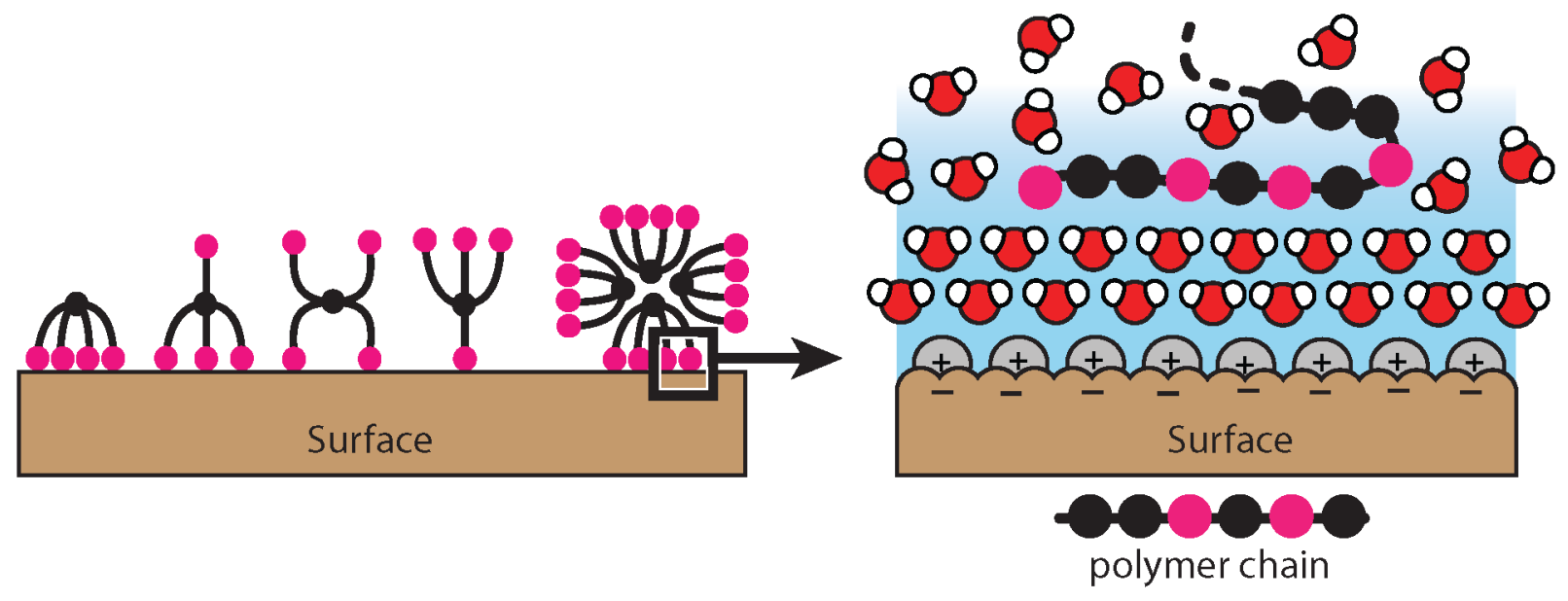




\section{Supporting information}

\section{Interaction Forces Between Pegylated Star-Shaped}

\section{Polymers at Mica Surfaces}

Xavier Banquy ${ }^{1}$, Frantz Le Dévédec ${ }^{2} \dagger$, Hsiu-Wei Cheng ${ }^{3,4}$, Jimmy Faivre ${ }^{1}$, Julian X.X. Zhu ${ }^{2}$, Markus Valtiner ${ }^{3,4}$

${ }^{1}$ Faculty of Pharmacy, Université de Montréal, C.P. 6128, Succursale Centre-ville, Montréal, QC H3C 3J7, Canada

${ }^{2}$ Department of Chemistry, Université de Montréal, C.P. 6128, Succursale Centre-ville, Montréal, QC H3C 3J7, Canada

${ }^{3}$ Interface Chemistry and Surface Engineering Max-Planck-Institut fír Eisenforschung MaxPlanck-Straße 1, 40237 Dísseldorf (Germany)

${ }^{4}$ Institut fir physikalische Chemie, TU Bergakademie Freiberg Leipziger Straße 29, 09599 Freiberg (Germany) 


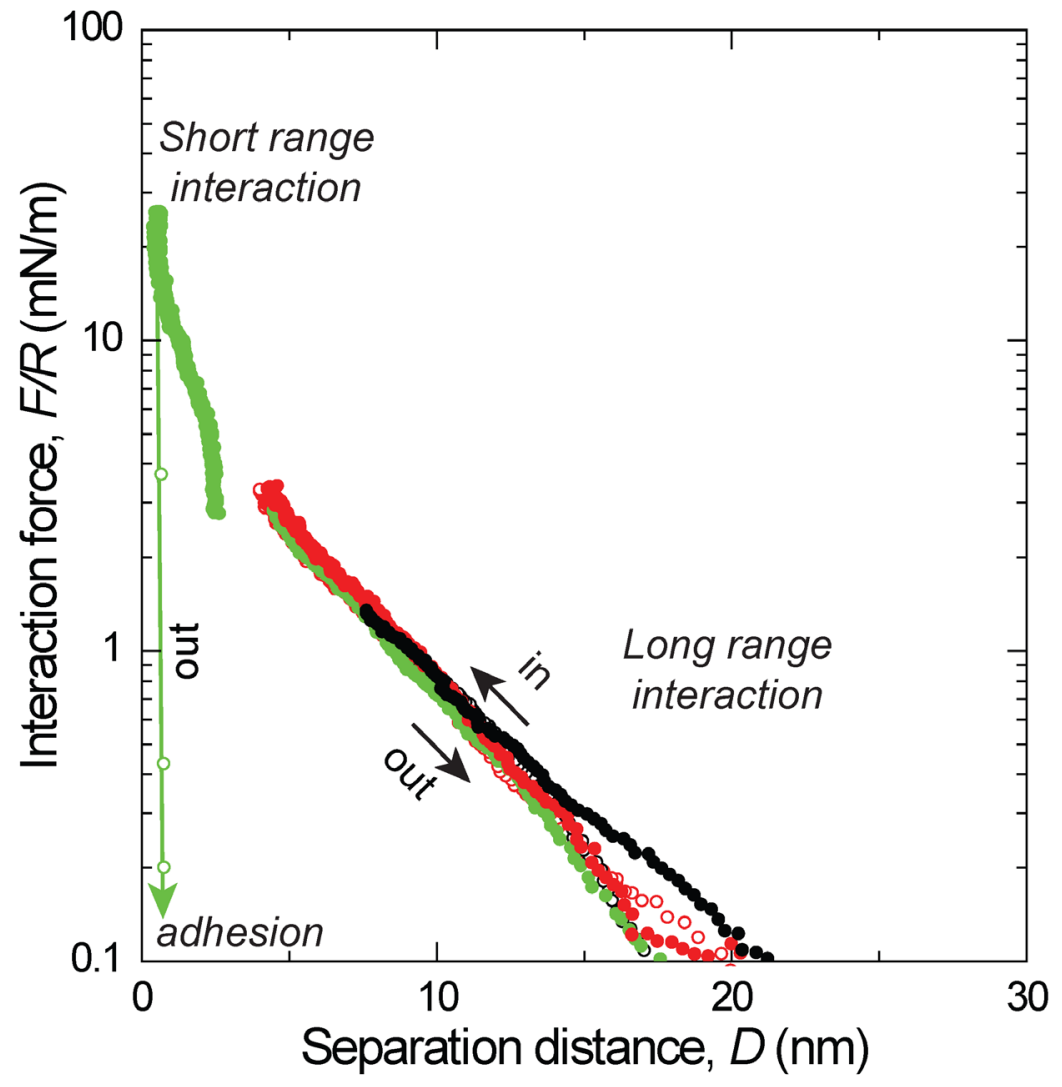

Fig. S1 Force profiles performed at different maximum applied force across a solution of $C A[E G 10 A G E 11 \mathrm{NH} 2] 4$ at $2 \mu \mathrm{g} / \mathrm{mL}$. In this graph, we can see that in the long range portion of the interaction profile, the in and out profiles are superimposed while once the short range force profile region is reached, strong adhesion is observed during the separation of the surfaces. Closed symbols correspond to in run profiles and open symbols to out run profiles. 